\title{
ARTICLE
}

Received 5 Jul 2014 | Accepted 9 Dec 2014 | Published 13 Jan $2015 \quad$ DOl: 10.1038/ncomms7056

\section{Observation of strong electron pairing on bands without Fermi surfaces in $\mathrm{LiFe}_{1-x} \mathrm{Co}_{x} \mathrm{As}$}

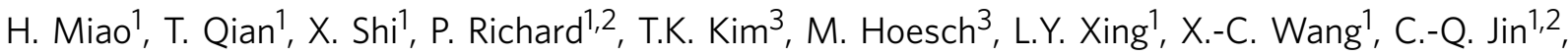 \\ J.-P. $H u^{1,2,4} \& H$. Ding ${ }^{1,2}$
}

In conventional BCS superconductors, the quantum condensation of superconducting electron pairs is understood as a Fermi surface instability, in which the low-energy electrons are paired by attractive interactions. Whether this explanation is still valid in high- $T_{c}$ superconductors such as cuprates and iron-based superconductors remains an open question. In particular, a fundamentally different picture of the electron pairs, which are believed to be formed locally by repulsive interactions, may prevail. Here we report a high-resolution angle-resolved photoemission spectroscopy study on $\mathrm{LiFe}_{1-x} \mathrm{CO}_{x} \mathrm{As}$. We reveal a large and robust superconducting gap on a band sinking below the Fermi level on Co substitution. The observed Fermi-surface-free superconducting order is also the largest over the momentum space, which rules out a proximity effect origin and indicates that the order parameter is not tied to the Fermi surface as a result of a surface instability.

\footnotetext{
${ }^{1}$ Beijing National Laboratory for Condensed Matter Physics, Institute of Physics, Chinese Academy of Sciences, Beijing 100190, China. ${ }^{2}$ Collaborative Innovation Center of Quantum Matter, Beijing 100190, China. ${ }^{3}$ Diamond Light Source, Harwell Campus, Didcot OX11 ODE, UK. ${ }^{4}$ Department of Physics, Purdue University, West Lafayette, Indiana 47907, USA. Correspondence and requests for materials should be addressed to T.Q. (email: taian@iphy.ac.cn) or to H.D. (dingh@iphy.ac.cn).
} 
$\mathrm{T}$ wo main categories of theoretical descriptions arise when trying to describe the high- $T_{\mathrm{c}}$ superconductivity of the iron-based superconductors (IBSCs): the weak coupling approach, which involves only the low-energy electronic structure near the Fermi level $\left(E_{\mathrm{F}}\right)$ (refs 1-5), and the strong coupling approach, which emphasizes the local magnetic moments and strong Coulomb interactions $s^{6-10}$. In the former, superconductivity emerges as a Fermi surface (FS) instability and is, in principle, sensitive to FS changes. In particular, the superconducting (SC) gap is tied to the FS and its amplitude is strongly influenced by the nesting conditions. In the latter, the pairing is caused by local antiferromagnetic exchange couplings, well defined in the real space, which lead to a SC order parameter (OP) that is fixed in the momentum space and relatively insensitive to small changes of the electronic structure near the FS.

In principle, one can distinguish between these two approaches and get critical information on the pairing mechanism of IBSCs by tracking precisely the evolution of the SC OP on bands for which the contributions to the FS vary drastically. In this respect, $\mathrm{LiFe}_{1-x} \mathrm{Co}_{x} \mathrm{As}$ offers a perfect platform for this study because it undergoes a Lifshitz transition with one FS disappearing at small Co substitution ${ }^{11,12}$.

Here we show a high-resolution angle-resolved photoemission spectroscopy (ARPES) study on SC $\mathrm{LiFe}_{1-x} \mathrm{Co}_{x}$ As. We reveal a large and robust SC gap with $2 \Delta_{\alpha} / k_{\mathrm{B}} T_{\mathrm{c}} \sim 7$ on the inner hole band that is sinking below the Fermi level on Co substitution. The observed FS-free SC gap is also the largest over the momentum space, which rules out a proximity effect origin and indicates that the SC OP is not tied to the FS as a result of a FS instability.

\section{Results}

FS topology of pristine $\mathrm{LiFeAs}$ and $\mathrm{LiFe}_{0.97} \mathrm{Co}_{0.03} \mathrm{As}$. We first look at the FS topologies of pristine $\mathrm{LiFeAs}$ and $\mathrm{LiFe}_{0.97} \mathrm{Co}_{0.03} \mathrm{As}$,
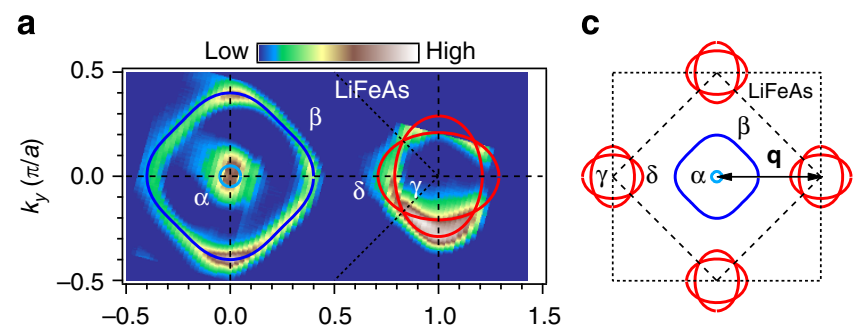

b

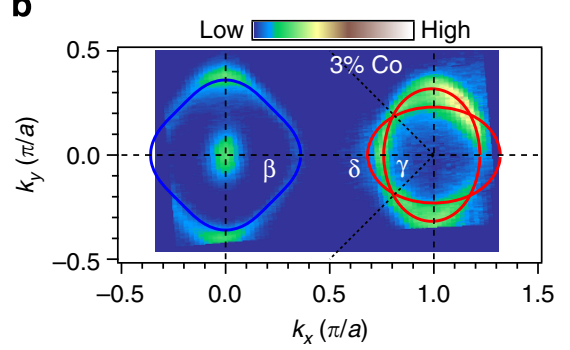

d

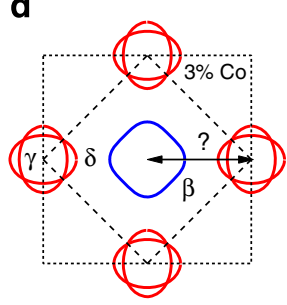

Figure 1 | FS topology of pristine LiFeAs and $\mathrm{LiFe}_{\mathbf{0 . 9 7}} \mathrm{Co}_{\mathbf{0 . 0 3}} \mathrm{As}$. (a,b) Plots of the ARPES intensity at $E_{\mathrm{F}}$ of LiFeAs $\left(T_{\mathrm{c}}=18 \mathrm{~K}\right)$ and LiFe ${ }_{0.97} \mathrm{Co}_{0.03} \mathrm{As}$ $\left(T_{\mathrm{c}}=15 \mathrm{~K}\right)$ as a function of the two-dimensional wavevector measured at $30 \mathrm{~K}$ with the $\mathrm{He} \mathrm{l} \alpha(h v=21.218 \mathrm{eV})$. The intensity is obtained by integrating the spectra within $10 \mathrm{meV}$ with respect to $E_{\mathrm{F} .}(\mathbf{c}, \mathbf{d})$ Extracted $k_{\mathrm{F}}$ loci of $\mathrm{LiFeAs}$ and $\mathrm{LiFe} \mathrm{F}_{0.97} \mathrm{CO}_{0.03} \mathrm{As}$, respectively. The small hole-like $\mathrm{FS}$ at the $B Z$ centre is sinking below $E_{F}$ due to the Co substitution and expected to significantly suppress the interband scattering between the electron and hole FSs. which are illustrated in Fig. 1. In agreement with previous studies, the substitution of Co introduces electron carriers and effectively moves the chemical potential upwards ${ }^{11-14}$. Since the inner holelike FS (the $\alpha$ FS) shown in Fig. 1a only barely crosses $E_{\mathrm{F}}$ in pristine LiFeAs ${ }^{15,16}$, a slight substitution of Fe by Co removes this tiny FS pocket at the $\Gamma(0,0)$ point, and thus the system undergoes a Lifshitz transition ${ }^{12}$. The remnant intensity at $E_{\mathrm{F}}$ around $\Gamma$ in Fig. $1 \mathrm{~b}$ is attributed to the limited energy resolution setting $(\sim 14 \mathrm{meV})$ for this normal state (NS) measurement $(T=30 \mathrm{~K})$, which broadens the spectral width beyond $E_{\mathrm{F}}$.

Band dispersions of $\mathrm{LiFe}_{1-x} \mathrm{Co}_{x} \mathrm{As}$ near $E_{\mathrm{F}}$. To accurately determine the band top of the $\alpha$ band, we performed highresolution $(\sim 3 \mathrm{meV})$ ARPES measurements in the vicinity of $\Gamma$ for samples at three doping levels $(x=0,1 \%, 3 \%$, with onset $T_{\mathrm{c}} \approx 18,16,15 \mathrm{~K}$, correspondingly, as shown in Supplementary Figs 1 and 2). As seen in Fig. 2d-f, the band top shifts to 4 and $8 \mathrm{meV}$ below $E_{\mathrm{F}}$ at Co contents of 1 and $3 \%$, respectively. This shift is also clearly demonstrated by the energy distribution curves (EDCs) shown in Fig. 2m-o. While the low-energy quasiparticle peaks of pristine LiFeAs are clearly cutoff by the FermiDirac (FD) function, those of the 1\% Co and 3\% Co samples shift below $E_{\mathrm{F}}$ with small spectral weight at $E_{\mathrm{F}}$ due the finite peak width.

Although the electronic structure of $\mathrm{LiFe}_{1-x} \mathrm{Co}_{x}$ As is quite two dimensional, previous studies indicate that the $\alpha$ band has a small dispersion along $k_{z}$ (refs $11,12,16$ ). To quantify the $k_{z}$ effect of the $\alpha$ band and confirm that it does not cross $E_{\mathrm{F}}$ along $k_{\mathrm{z}}$, we extract the NS dispersion of the $\alpha$ band at $k_{z}=0$ and $\pi$. Figure $3 \mathrm{a}, \mathrm{c}$ shows the ARPES intensity plots of $3 \% \mathrm{Co}$ at $51 \mathrm{eV}\left(k_{z}=0\right)$ and $35 \mathrm{eV}$ $\left(k_{z}=\pi\right)$, respectively. The data are recorded at $20 \mathrm{~K}$ with linearly polarized light to enhance the intensity of the $\alpha$ band ${ }^{11,17}$. The intensity plots and EDCs show that the band top of the $\alpha$ band has tiny dispersion along $k_{z}$ and prove that at least for $3 \% \mathrm{Co}$, the $\alpha$ band is completely sinking below $E_{\mathrm{F}}$ all over the momentum space. The disappearance of the $\alpha$ FS reduces the density-of-states near $E_{\mathrm{F}}$ and hence significantly suppresses the interband scattering between the $\alpha$ band and the electron FSs at the M point $(\pi, 0)$, as seen from Fig. $2 \mathrm{~m}-\mathrm{o}$.

In the SC state, electrons are gapped towards higher binding energies and form a well-defined Bogoliubov quasiparticle peak. Figure $2 \mathrm{~m}$ compares the representative EDCs of pristine LiFeAs across the $\Gamma$ point above and below $T_{c}$. The electronic states within the SC gap are significantly altered, while the states at higher binding energies are only slightly modified by the Bogoliubov dispersion:

$$
E_{k}=\sqrt{\xi_{k}^{2}+\Delta_{k}^{2}}
$$

where $E_{k}$ is the energy of the Bogoliubov quasiparticle, which will not show clear deviation from the NS energy $\xi_{k}$ when $\xi_{k}$ is much larger than the SC gap $\Delta_{k}$. Interestingly, we find that near the band top, the electronic states of the $1 \%$ Co and 3\% Co samples are slightly shifted to higher binding energies as shown in Fig. 2n,o, indicating the opening of a SC gap on the $\alpha$ band, even though this $\alpha$ band is located below $E_{\mathrm{F}}$ at these doping levels (Supplementary Fig. 3).

Extraction of the SC gap. To see how the SC condensation affects the electronic states away from $E_{\mathrm{F}}$, we show the simulated EDCs without FD distribution in the NS and SC state in Fig. 4b, corresponding to the vertical line at the $\Gamma$ point in the simulated NS intensity plot in Fig. 4a. The band tops are set at 8 and $20 \mathrm{meV}$ below $E_{\mathrm{F}}$, respectively, in agreement with the real band positions in this material. With a $5-\mathrm{meV}$ SC gap turned on, the low-energy peak (P1) is shifted by $2 \mathrm{meV}$, while the high-energy peak (P2) is 


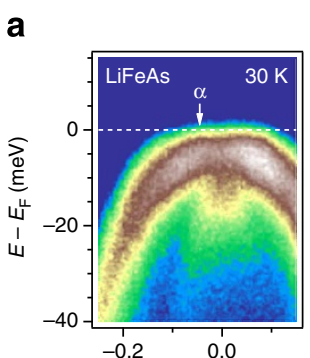

d

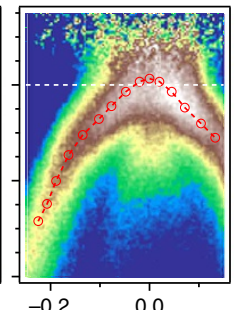

b

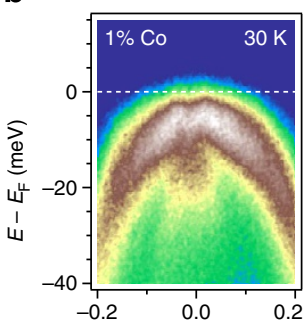

e
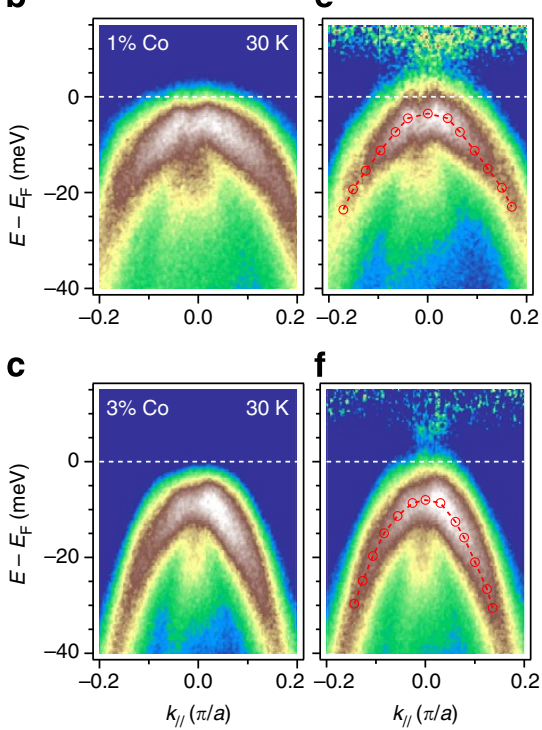

C $f$

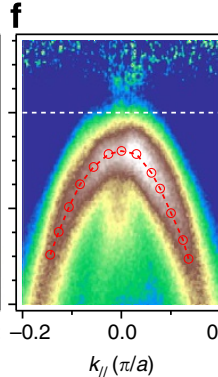

g

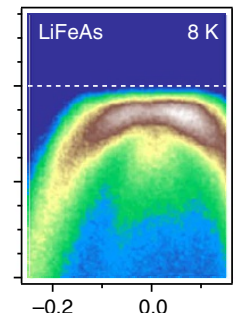

h

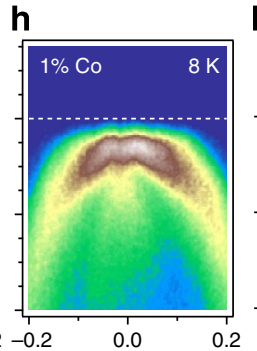

i

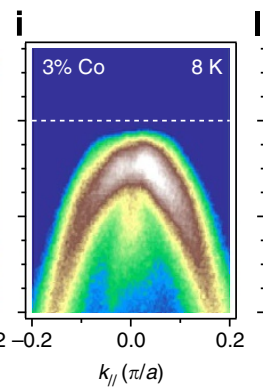

j

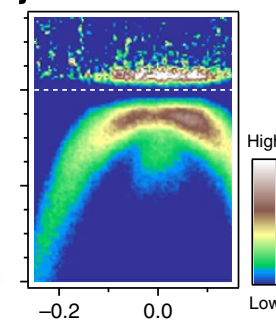

k

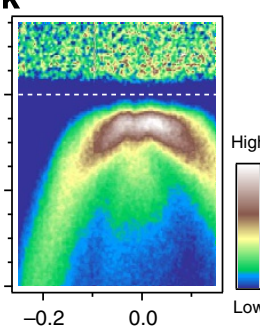

I

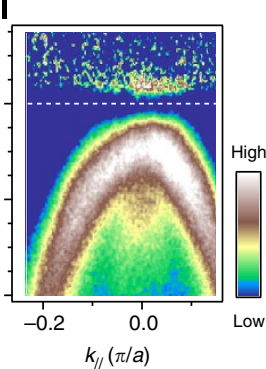

m

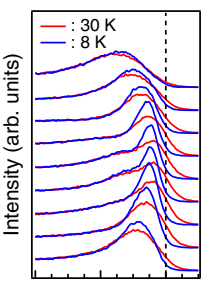

n

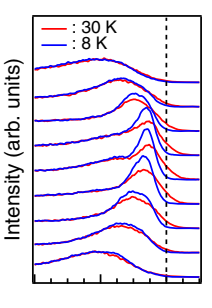

0

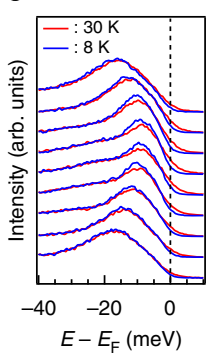

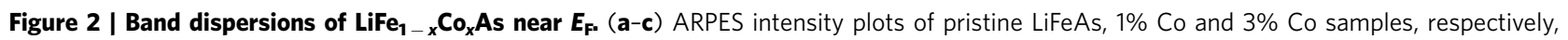
in the NS across the BZ centre. The data are recorded with the $\mathrm{He} l \alpha$ line $(h v=21.218 \mathrm{eV})$, which is close to $k_{z}=0$. (d-f) Same data as in a-c, but divided by the FD function convoluted with the system resolution. Red circles are the extracted NS dispersion of the $\alpha$ band. The $\alpha$ band is sinking below $E_{F}$ in the $1 \%$ Co and $3 \%$ Co compounds. ( $\mathbf{g}-\mathbf{i}$ ) Corresponding intensity plots in the SC state. (j-I) Same data as in $\mathbf{g}$-i, but divided by the FD function convoluted with the system resolution. The difference between the NS and the SC state is clearly resolved (m-o), where the representative EDCs in both the NS and the SC state are plotted together. The enhanced spectral weight at the low binding energy is attributed to the coherence of the paired electrons.

a

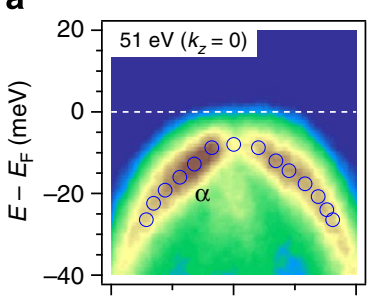

b

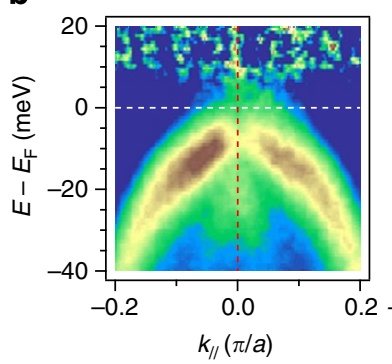

C

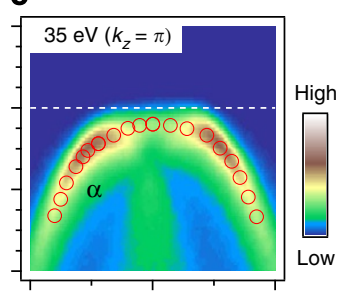

d

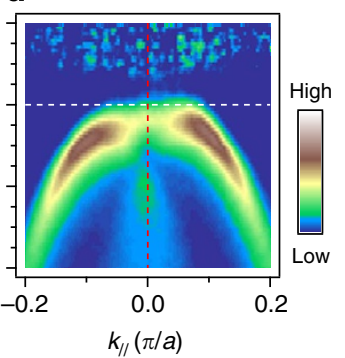

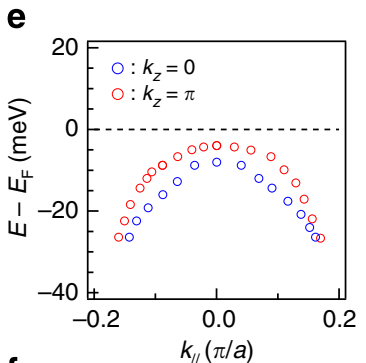

$\mathbf{f}$

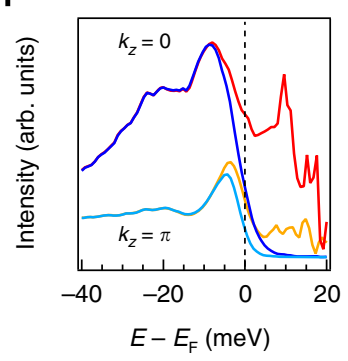

Figure 3 | Band dispersion of the $\boldsymbol{\alpha}$ band at $\boldsymbol{k}_{\mathbf{z}}=\mathbf{O}$ and $\boldsymbol{\pi}$ in LiFe $\mathbf{~}_{\mathbf{0 . 9 7}} \mathbf{C o}_{\mathbf{0 . 0 3}}$ As. (a,c) show the ARPES intensity plots of $\mathrm{LiFe}_{0.97} \mathrm{Co}_{0.03} \mathrm{As}$ at $51 \mathrm{eV}$ $\left(k_{z}=0\right)$ and $35 \mathrm{eV}\left(k_{z}=\pi\right)$, respectively. The data are recorded at $20 \mathrm{~K}$ with linearly polarized light to enhance the $\alpha$ band $d^{11,17}$. Blue and red circles on top of the intensity are extracted from EMDCs. (b,d) are the same data but divided by the FD function convoluted with the system resolution. (e) The extracted band dispersions from a,c are plotted together and prove that the band top of the $\alpha$ band has tiny dispersion along $k_{z}$. (f) The EDCs at the Brillouin zone centre as marked by the red dashed line in $\mathbf{b}, \mathbf{d}$. Red and yellow curves are the same data of blue and cyan curves but divided by the FD function convoluted with the system resolution. 

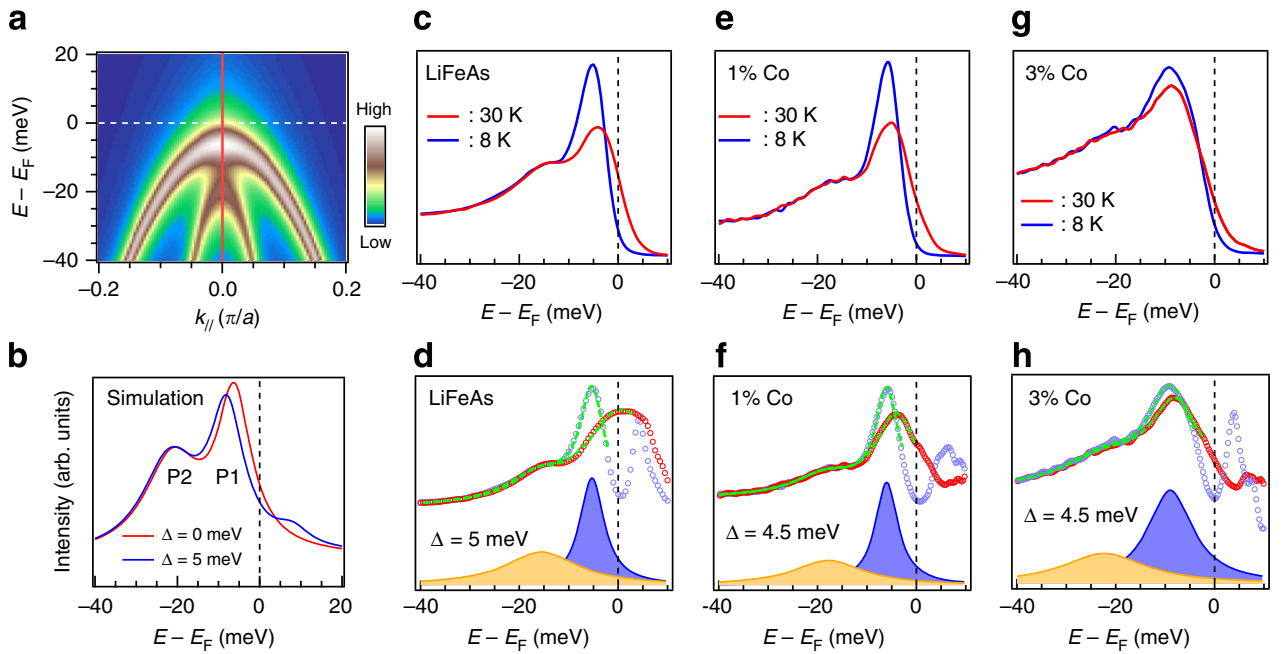

Figure 4 | Extracting the SC gap from EDCs. (a) ARPES intensity plot that simulates the NS band dispersion without FD distribution of $3 \%$ Co sample. (b) Simulated EDCs in the NS (red) and SC state (blue), corresponding to the vertical line in the intensity plot in a. The spectral function is assumed to have the BCS form. The small peak above $E_{\mathrm{F}}$ in the blue EDC is due to the particle-hole mixing, which is a hallmark of SC condensation. By using the BCS spectral function to fit the EDCs in the NS and the SC state, we extract the SC gap. (c,e,g) EDCs at the BZ centre in the NS and the SC state of pristine LiFeAs, 1\% Co and 3\% Co samples, respectively. The EDCs are obtained by combining three EDCs near the Brillouin zone centre. To extract the SC gap, the raw data shown in $\mathbf{c}, \mathbf{e}, \mathbf{g}$ are divided by FD function convoluted with the system resolution and shown in $\mathbf{d}, \mathbf{f}, \mathbf{h}$, respectively. Green dotted curves on light blue and red circles are the fitting results. The decomposed spectral functions in the SC state are appended below the fittings. The broadening of the decomposed peaks at higher doping levels is likely caused by an enhanced impurity scattering due to the in-plane substitution.

almost unchanged. Moreover, due to the particle-hole mixing, a small peak above $E_{\mathrm{F}}$ develops in the EDC of the SC state. Back to the experimental data, in Fig. 4c,e,g, the EDCs at the BZ centre exhibit a coherent peak developing at all three doping levels below $T_{c}$. Figure $4 \mathrm{~d}, \mathrm{f}, \mathrm{h}$ shows the same data but divided by the FD function convoluted with the system resolution. To extract the SC gap, the EDCs in the NS and SC state are fitted by the BCS spectral function plus a constant background (Supplementary Table 1). The extracted SC gap keeps almost constant, while the line width of the coherent peak becomes broader at higher doping levels. This indicates that the Co substitution introduces impurity potentials, which is believed to play a destructive role in sign-reversal pairing, at least in the weak coupling regime ${ }^{1}$.

Following the procedure shown in Fig. 4, we extract the lowenergy band dispersion below and above $T_{\mathrm{c}}$. Figure $5 \mathrm{a}-\mathrm{d}$ show the extracted data of the $1 \%$ Co and 3\% Co samples in wide and narrow energy ranges. In agreement with equation (1), the band shift is the largest near the band top and quickly vanishes at higher binding energies. By using the NS data to fit the dispersion in the SC state, we extract the SC gaps of pristine LiFeAs, $1 \%$ Co and $3 \%$ Co and plot them as a function of the Co concentration in Fig. 5e. The SC gap on the $\alpha$ band remains almost constant, while the associated FS topology undergoes a Lifshitz transition with the substitution of Co.

Since $\mathrm{LiFe}_{1-x} \mathrm{Co}_{x} \mathrm{As}$ is a multi-orbital superconductor, the large SC gap observed on the $\alpha$ band, in principle, can be induced via proximity to other bands, which are crossing $E_{\mathrm{F}}$ at all doping levels. In this scenario, the SC gap on the $\alpha$ band is expected to be smaller than the other bands and to give a negligible contribution to the SC pairing. To justify this point, we show the SC gap on the $\beta, \gamma$ and $\delta$ bands of $3 \%$ Co in Fig. 6. The extracted SC gap on the $\beta, \delta$ and $\gamma$ bands are 3.3, 3.5 and $4 \mathrm{meV}$, respectively (Supplementary Table 2). Since the cut on the $\gamma$ band measures the largest gap on the electron FSs ${ }^{15,16}$, our results prove that the SC gap on the $\alpha$ band is the largest over the momentum space and thus rule out the possibility of a proximity effect causing by the pairing on other FS sheets.

\section{Discussion}

As discussed before, the interband scattering in the particleparticle channel is dramatically reduced due to the Lifshitz transition. According to the weak coupling nesting scenarios, the SC gap on the $\alpha$ band is expected to exponentially decrease when the contribution of this band to the density-of-states at $E_{\mathrm{F}}$ goes to zero ${ }^{18,19}$, as shown in Fig. 5e. This is clearly in contradiction with our experimental observation that the SC gap on the $\alpha$ band is robust and the largest over the momentum space.

Recent inelastic neutron scattering and NMR studies have demonstrated that low-energy spin fluctuations are relatively weak and incommensurate for pristine $\mathrm{LiFeAs}{ }^{20-22}$. Combined with ARPES data, the incommensurate inelastic neutron scattering peaks were attributed to the interband scattering between the $\beta$ FS and the electron $\mathrm{FSs}^{23}$. However, ARPES and STM studies demonstrate that the largest SC gap is indeed on the $\alpha$ band, which is clearly incompatible with the low-energy spin fluctuations scenario ${ }^{15,16,24,25}$. Although this discrepancy can be removed by subtle modifications involving orbital fluctuations or small-q interband scattering 26,27 , our observation of strong SC pairing on the band without FS is beyond any reasonable mending within the weak coupling approach. Instead, it is naturally consistent with many strong coupling approaches ${ }^{6-10,28,29}$, in particular with the $\mathrm{J}_{1}-\mathrm{J}_{2}$ model that predicts the strongest pairing at the zone centre ${ }^{6-9}$. Indeed, the ratio $2 \Delta_{\alpha} / k_{\mathrm{B}} T_{\mathrm{c}} \sim 7$ observed in the $\mathrm{LiFe}_{1-x} \mathrm{Co}_{x}$ As is twice larger than the predicted BCS value, which also suggests a strong coupling pairing mechanism.

Finally, it is interesting to compare the observed SC gap with $\varepsilon_{\mathrm{F}}$, here defined as the energy difference from the band top to the chemical potential ${ }^{30}$, as illustrated in Fig. 5a. Previous studies on the iron-chalcogenide superconductor $\mathrm{FeTe}_{1-} \mathrm{Se}_{x}$ (refs 30,31) show that the SC gap is comparable with $\varepsilon_{\mathrm{F}}$ and consistent with a BCS-BEC crossover scenario ${ }^{29,30}$. In $\mathrm{LiFe}_{1-x} \mathrm{Co}_{x} \mathrm{As}$, as shown in Fig. $5 \mathrm{e}$, the value of $\varepsilon_{\mathrm{F}}$ drops from +2 to $-8 \mathrm{meV}$ with $3 \% \mathrm{Co}$ substitution, while the SC gap remains almost unchanged and is 

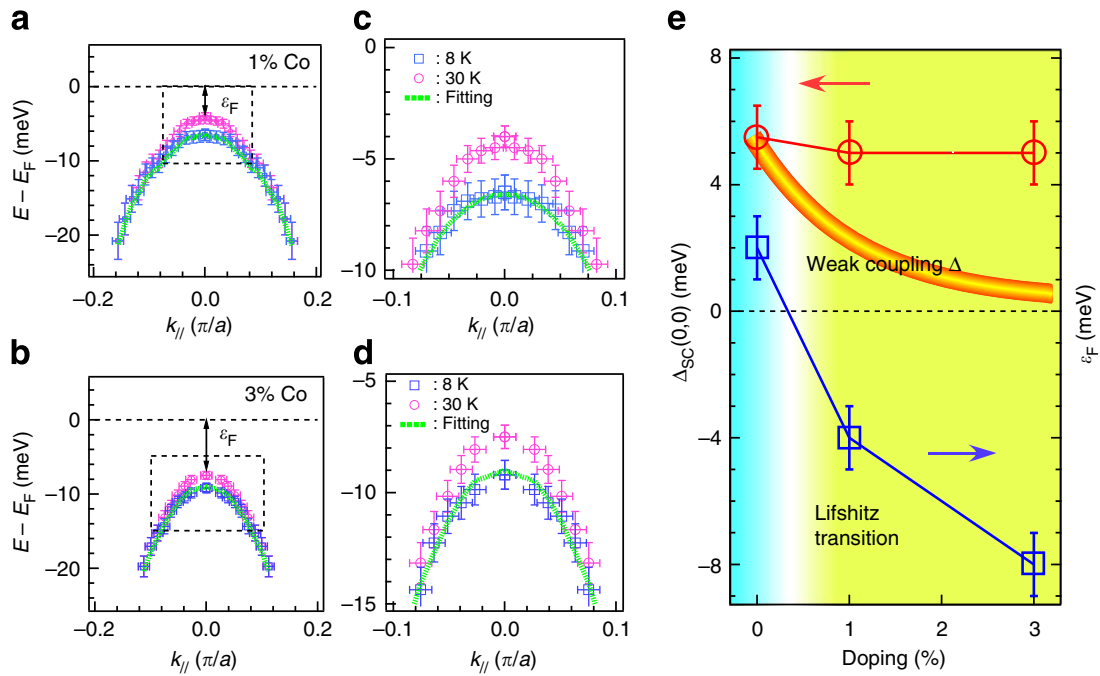

Figure 5 | Extraction of the SC gap from dispersions and robustness of the pairing against the disappearance of FS. (a,b) Extracted band dispersions of $1 \% \mathrm{Co}$ and 3\% Co below (light blue squares) and above (pink circles) $T_{\mathrm{c}}$. The energy error bars are determined by the s.d. of the fitted parameters. The momentum error bars are determined by our momentum resolution times a factor of 3 , since we combined three nearby EDCs to reduce the noise level. A zoom into a narrower energy range in $\mathbf{c}, \mathbf{d}$ show that the difference between the NS and the SC state is the largest at the BZ centre, and then gets smaller at high binding energies. The green dotted curves are the fitting results, which yield a similar SC gap as extracted from EDCs. (e) Comparison of the extracted SC gap from the experimental data and the expected ones from weak coupling theories. This shows that the SC gap observed on the $\alpha$ band is robust despite the disappearance of the $\alpha$ FS at the $\Gamma$ point. This observation is incompatible with the weak coupling theory, in which the SC gap is expected to be sensitive to both the density-of-states near $E_{\mathrm{F}}$ and impurity scattering.

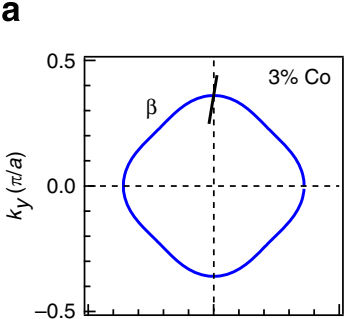

b

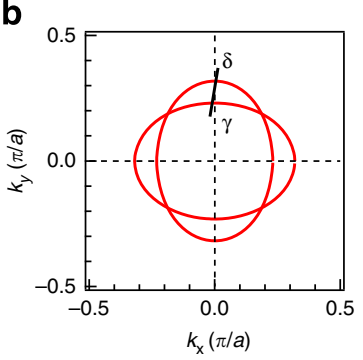

\section{C}

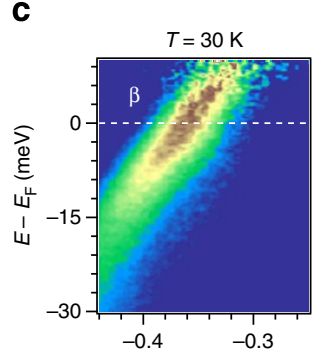

d

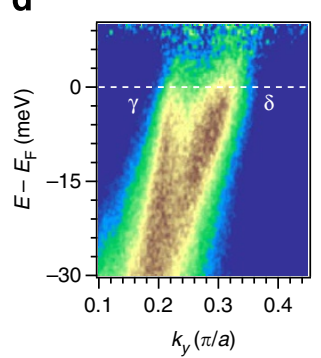

e

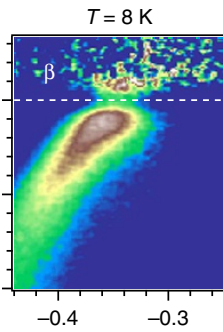

f

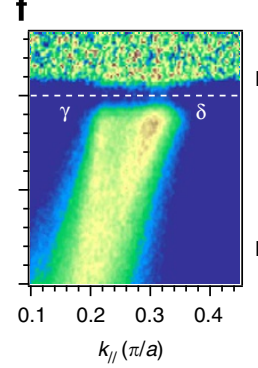

g

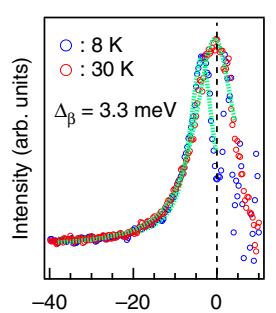

h

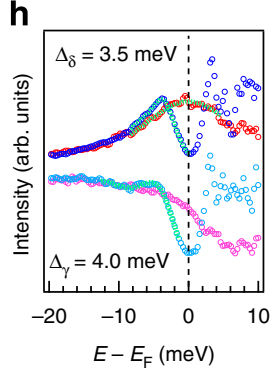

Figure 6 | SC gap on $\boldsymbol{\beta}$ and electron FSs. The measured $k_{F}$ positions are illustrated in $\mathbf{a}$ and $\mathbf{b}$. (c-f) ARPES intensities that are divided by the FD function convoluted with the system resolution. NS and SC state EDCs that are crossed the $k_{\mathrm{F}}$ positions of the $\beta, \gamma$ and $\delta$ bands are shown in $\mathbf{g}$ and $\mathbf{h}$. Red/pink and blue/cyan circles represent the NS and SC state EDCs respectively. The gap values are extracted by using BCS spectral function plus a polynomial function. The fitted results are shown in $\mathbf{g}$ and $\mathbf{h}$ with green dashed curves.

immune to the Lifshitz transition. We note that due to the multi-orbital nature of the IBSCs, the value of the Fermi energy might be much larger than we defined here, and whether the $\mathrm{LiFe}_{1-x} \mathrm{Co}_{x}$ As lays in the BCS-BEC crossover regime needs further experimental and theoretical investigations.

\section{Methods}

Sample preparation and experimental setup. Single crystals of $\mathrm{LiFe}_{1-x} \mathrm{Co}_{x} \mathrm{As}$ were synthesized by the self-flux method using $\mathrm{Li}_{3} \mathrm{As}, \mathrm{Fe}_{1-x} \mathrm{Co}_{x} \mathrm{As}$ and As powders as starting materials. The $\mathrm{Li}_{3} \mathrm{As}, \mathrm{Fe}_{1-} \mathrm{Co}_{x} \mathrm{As}$ and As powders were weighed according to the element ratio of $\mathrm{Li}\left(\mathrm{Fe}_{1-x} \mathrm{Co}_{x}\right)_{0.3}$ As. The mixture was grounded and put into an alumina crucible and sealed in $\mathrm{Nb}$ crucibles under 1 atm of Argon gas. The $\mathrm{Nb}$ crucible was then sealed in an evacuated quartz tube, heated to $1,100^{\circ} \mathrm{C}$ and slowly cooled down to $700^{\circ} \mathrm{C}$ at a rate of $3^{\circ} \mathrm{C}$ per hour. High-energyresolution ARPES data were recorded at the Institute of Physics, Chinese Academy of Sciences, using the He I $\alpha(h v=21.218 \mathrm{eV})$ resonance line of a helium discharge lamp. The angular and momentum resolutions were set to $0.2^{\circ}$ and $3 \mathrm{meV}$, respectively. ARPES polarization-dependent measurements were performed at Beamline I05 of Diamond Light Source using a Scienta R4000 with energy and momentum resolutions set to $0.2^{\circ}$ and $10 \mathrm{meV}$, respectively. To select the $\alpha$ band, we employed linearly polarized light with the potential vector perpendicular to the mirror plane of the sample. All samples were cleaved in situ. The data were taken in a vacuum better than $3 \times 10^{-11}$ Torr with discharge lamp and $1 \times 10^{-10}$ Torr with synchrotron light source. 
Extraction of the SC gap. To extract the SC gap from EDCs, we use the functions:

$$
\begin{gathered}
I(\omega)=C_{0}+\frac{a_{1}}{\left(\left(\omega-E_{k}^{1}\right)^{2}+\Gamma_{1}^{2}\right)}+\frac{a_{2}}{\left(\left(\omega-E_{k}^{2}\right)^{2}+\Gamma_{2}^{2}\right)} \\
I(\omega)=C_{0}+\frac{a}{\left(\left(\omega-E_{k}\right)^{2}+\Gamma^{2}\right)} \\
I(\omega)=C_{0}+C_{1}\left(\frac{\omega}{10}\right)^{2}+C_{2}\left(\frac{\omega}{10}\right)^{4}+\frac{a}{\left(\left(\omega-E_{k}\right)^{2}+\Gamma^{2}\right)} \\
E_{k}^{i}=\sqrt{\left(\left(\xi_{k}^{i}\right)^{2}+\Delta_{k}^{2}\right)} \\
a_{i} \propto \Gamma_{i}\left(u_{k}^{i}\right)^{2}=\frac{1}{2} \Gamma_{i}\left(1+\frac{\xi_{k}^{i}}{E_{k}^{i}}\right)
\end{gathered}
$$

Here we assume a BCS spectral function in the occupied states, with the $\Gamma_{k}$ parameter not changing with binding energy. $a_{i}$ is a fitting constant, which is proportional to $\Gamma_{k}$ and $u_{k}^{2}$. The fitting parameters are summarized in Supplementary Tables 1 and 2. Equations (2)-(4) are used to fit the SC gap of the $\alpha$, $\beta$ and $\gamma / \delta$ FSs, respectively. The first and second BCS spectral function in equation (2) are used to fit the $\alpha$ band and $\alpha^{\prime}$ band, respectively ${ }^{11}$. Since the $\gamma$ and $\delta$ bands are close to each other, we use a polynomial function to remove the large background, and we assume the background has an even symmetry with respect to $E_{\mathrm{F}}$ (ref. 32). Since the BCS spectral function can be derived from the Bogoliubov formalism, it can be applied to the strong coupling regime $e^{31,33-35}$.

\section{References}

1. Hirschfeld, P. J., Korshunov, M. M. \& Mazin, I. I. Gap symmetry and structure of Fe-based superconductors. Rep. Prog. Phys. 74, 124508 (2011).

2. Scalapino, D. J. A common thread: the pairing interaction for unconventional superconductors. Rev. Mod. Phys. 84, 1383 (2012).

3. Kuroki, K. et al. Unconventional pairing originating from the disconnected Fermi surfaces of superconducting $\mathrm{LaFeAsO}_{1-x} \mathrm{~F}_{x}$. Phys. Rev. Lett. 101, 087004 (2008).

4. Mazin, I. I, Singh, D. J., Johannes, M. D. \& Du, M. H. Unconventional superconductivity with a sigh reversal in the order parameter of $\mathrm{LaFeAsO}_{1-x} \mathrm{~F}_{x}$. Phys. Rev. Lett. 101, 057003 (2008).

5. Kontani, H. \& Onari, S. Orbital-fluctuation-mediated superconductivity in iron pnictides: analysis of the five-orbital Hubbard-Holstein model. Phys. Rev. Lett. 104, 157001 (2010).

6. Seo, K., Bernevig, B. A. \& Hu, J. P. Pairing symmetry in a two-orbital exchange coupling model of oxypnictides. Phys. Rev. Lett. 101, 206404 (2008).

7. Si, Q \& Abrahams, E. Strong correlations and magnetic frustration in the high Tc iron pnictides. Phys. Rev. Lett. 101, 076401 (2008).

8. Yildirim, T. Origin of the $150 \mathrm{~K}$ anomaly in LaFeAsO: competing antiferromagnetic interactions, frustration, and a structural phase transition. Phys. Rev. Lett. 101, 057010 (2008).

9. Hu, J. P. \& Ding, H. Local antiferromagnetic exchange and collaborative Fermi surface as key ingredients of high temperature superconductors. Sci. Rep. 2, 381 (2012).

10. Davis, J. C. S. \& Lee, D.-H. Concepts relating magnetic interactions, intertwined electronic orders, and strongly correlated superconductivity. Proc. Natl Acad. Sci. USA 110, 17623-17630 (2013).

11. Miao, H. et al. Coexistence of orbital degeneracy lifting and superconductivity in iron-based superconductors. Phys. Rev. B. 89, 220503 (2014).

12. Ye, Z.-R. et al. Extraordinary quasiparticle scattering and bandwidth-control by dopants in iron-based superconductors. Phys. Rev. X 4, 031041 (2014).

13. Berlijn, T., Lin, C. -H., Garber, W. \& Ku, W. Do transition-metal substitutions dope carriers in iron-based superconductors? Phys. Rev. Lett. 108, 207003 (2012).

14. Ideta, S. et al. Dependence of carrier doping on the impurity potential in transition-metal-substituted FeAs-based superconductors. Phys. Rev. Lett. 110, 107007 (2013)

15. Umezawa, K. et al. Unconventional anisotropic s-wave superconducting gaps of the LiFeAs iron-pnictide superconductors. Phys. Rev. Lett. 108, 037002 (2012).

16. Borisenko, S. V. et al. One-sign order parameter in iron based superconductor. Symmetry 4, 251-264 (2012).

17. Wang, X.-P. et al. Orbital characters determined from Fermi surface intensity patterns using angle-resolved photoemission spectroscopy. Phys. Rev. B 85, 214518 (2012)
18. $\mathrm{Xu}, \mathrm{N}$. et al. Possible nodal superconducting gap and Lifshitz transition in heavily hole-doped $\mathrm{Ba}_{0.1} \mathrm{~K}_{0.9} \mathrm{Fe}_{2} \mathrm{As}_{2}$. Phys. Rev. B 88, 220508(R) (2013).

19. Yin, Z. P., Haule, K. \& Kotliar, G. Spin dynamics and orbital-antiphase pairing symmetry in iron-based superconductors. Nat. Phys. 10, 845-850 (2014).

20. Taylor, A. E. et al. Antiferromagnetic spin fluctuations in LiFeAs observed by neutron scattering. Phys. Rev. B. 83, 220514(R) (2011).

21. Ma, L., Zhang, J., Chen, G. F. \& Yu, W. NMR evidence of strongly correlated superconductivity in LiFeAs: tuning toward a spin-density-wave ordering. Phys. Rev. B. 82, 180501(R) (2010).

22. Wang, M. et al. Effect of Li-deficiency impurities on the electron-overdoped LiFeAs superconductor. Phys. Rev. B. 86, 144511 (2012).

23. Knolle, J. et al. Incommensurate magnetic fluctuations and Fermi surface topology in LiFeAs. Phys. Rev. B. 86, 174519 (2012).

24. Allan, M. P. et al. Anisotropic energy gaps of iron-based superconductivity from intraband quasiparticle interference in LiFeAs. Science 336, 563-567 (2012).

25. Wang, Y. et al. Superconducting gap in LiFeAs from three-dimensional spinfluctuation pairing calculations. Phys. Rev. B. 88, 174516 (2013).

26. Ahn, F. et al. Superconductivity from repulsion in LiFeAs: novel s-wave symmetry and potential time-reversal symmetry breaking. Phys. Rev. B 89, 144513 (2014)

27. Saito, T. et al. Reproduction of experimental gap structure in LiFeAs based on the orbital-spin fluctuation theory: $\mathrm{s}_{++}$-wave, $\mathrm{s}_{ \pm}$-wave, and $\mathrm{s}^{\mathrm{h}} \pm$-wave states. Phys. Rev. B 90, 035102 (2014).

28. You, Y.-Z., Yang, F., Kou, S.-P. \& Weng, Z.-Y. Phase diagram and a possible unified description of intercalated iron selenide superconductors. Phys. Rev. Lett. 107, 167001 (2011).

29. Guidini, A. \& Perali, A. Band-edge BCS-BEC crossover in a two-band superconductor: physical properties and detection parameters. Supercond. Sci. Technol. 27, 124002 (2014).

30. Lubashevsky, Y. et al. Shallow pockets and very strong coupling superconductivity in $\mathrm{FeSe}_{x} \mathrm{Te}_{1-x}$. Nat. Phys. 8, 309-312 (2012).

31. Miao, H. et al. Isotropic superconducting gaps with enhanced pairing on electron Fermi surfaces in $\mathrm{FeTe}_{0.55} \mathrm{Se}_{0.45}$. Phys. Rev. B 85, 094506 (2012).

32. Liu, Z.-H. et al. Unconventional superconducting gap in $\mathrm{NaFe}_{0.95} \mathrm{Co}_{0.05} \mathrm{As}$ observed by angle-resolved photoemission spectroscopy. Phys. Rev. B 84, 064519 (2011).

33. Matsui, H. et al. BCS-like Bogoliubov quasiparticles in high- $T_{\mathrm{c}}$ superconductors observed by angle-resolved photoemission spectroscopy. Phys. Rev. Lett. 90 , 217002 (2003).

34. Ding, H. et al. Observation of Fermi-surface-dependent nodeless superconducting gaps in $\mathrm{Ba}_{0.6} \mathrm{~K}_{0.4} \mathrm{Fe}_{2} \mathrm{As}_{2}$. Europhys. Lett. 83, 47001 (2008).

35. Chen, Q., Stajic, J., Tan, S. \& Levin, K. BCS-BEC crossover: from high temperature superconductors to ultracold superfluids. Phys. Rep. 412, 1-88 (2005).

\section{Acknowledgements}

We thank A. Chubukov, Y.M. Dai, W. Ku and X.R. Liu for useful discussions. This work was supported by grants from CAS (2010Y1JB6 and XDB07000000), MOST (2010CB923000, 2011CBA001000 and 2013CB921700) and NSFC (11234014 and 11274362).

\section{Author contributions}

H.M. and H.D. designed the experiments; H.M., T.Q., X.S., P.R., T.K.K. and M.H. carried out the experiments; H.M. analysed the data; L.Y.X., X.-C.W. and C.-Q.J. provided the samples; H.M., T.Q., P.R., J.-P.H. and H.D. wrote the manuscript. All authors discussed the results and commented on the manuscript.

\section{Additional information}

Supplementary Information accompanies this paper at http://www.nature.com/ naturecommunications

Competing financial interests: The authors declare no competing financial interests.

Reprints and permission information is available online at http://npg.nature.com/ reprintsandpermissions/

How to cite this article: Miao, H. et al. Observation of strong electron pairing on bands without Fermi surfaces in $\mathrm{LiFe}_{1-x} \mathrm{Co}_{x}$ As. Nat. Commun. 6:6056 doi: 10.1038/ncomms7056 (2015). 